\title{
Exploring New Socio-Economic Thoughts for a Small and Narrow Earth
}

\author{
Mamoru Kaneko \\ Faculty of Political Science and Economics, Waseda University, Tokyo, Japan \\ Email: mkanekoepi@waseda.jp
}

How to cite this paper: Kaneko, M (2018). Exploring New Socio-Economic Thoughts for a Small and Narrow Earth. Advances in Applied Sociology, 8, 397-421. https://doi.org/10.4236/aasoci.2018.85024

Received: January 23, 2018

Accepted: May 12, 2018

Published: May 15, 2018

Copyright $\odot 2018$ by author and Scientific Research Publishing Inc. This work is licensed under the Creative Commons Attribution International License (CC BY 4.0).

http://creativecommons.org/licenses/by/4.0/

\begin{abstract}
The earth has been getting smaller and narrower with the expansion of human activities. Now, it is an urgent task for social scientists to explore and study new socio-economic thoughts for this small and narrow earth. This article provides a normative theory and a descriptive theory for the present and future earth. The former is to provide, viewing the earth and human community as unity, evaluations of possible events and of designs of social institutions. The normative theory helps us think about where we should direct the earth. The latter discusses social sciences for practical management of the earth. Since, however, great diversity of cultures will remain, a unified management of the earth is practically impossible. We are required to rethink and develop new socio-economic thoughts in radical manners.
\end{abstract}

\section{Keywords}

Free-Market Libertarian, Large-Environment, Small Earth, Normative

Theory, Nash Social Welfare, Descriptive Theory, Cognizance Assumption, Inductive Game Theory, Bounded Rationality

\section{Introduction}

As the century has turned into the 21st, we continue to witness poverty, famine, hungers, deadly diseases, conflicts, wars, genocides, and other serious problems in the world. These events have been problems at any time of our history, but they are now more worldwide as human economic activities with technological progress are getting larger and non-negligible relative to the size of the earth. These sizable impacts require us to rethink a new socio-economic thought and also to consider the management of the entire earth by taking the influences of human economic activities seriously into account. In this article, we explore what we, as social scientists, should and can do for the future earth. 
This article consists of two almost contradictory parts: a normative theory and a descriptive theory. The former is to provide, viewing the earth and human community as unity, evaluations of possible events and of designs of social institutions. The latter discusses social sciences for practical management of the earth, but at the same time, it shows that a great diversity of cultures remains and a unified management of the earth is practically impossible. Social problems and theoretical/methodological thoughts to be discussed vary over a vast range. It would be helpful to summarize the main themes of this article and to give a map of the subjects to be discussed.

The main themes are:

- small and narrow earth: unity of the earth vs. diverse cultures;

- dominant socio-economic thoughts;

- normative theory for the unity of the earth;

- human nature: bounded rationality and conservativeness;

- social scientific research on these problems;

- practical managements of the earth and societies.

Figure 1 depicts the logical relationships of these themes. The top box states the conflicting features of the present earth, which are discussed in Section 2. Then, we go to the normative theory, which is in the left box and discussed in Section 3. The right box consists of thoughts on the present economics and game theory, which are discussed in Section 4. Sections 5 and 6 address the necessities of descriptive/empirical studies and practical managements of the future earth.

Let us look at each section. In Section 2, we discuss conflicting features on dominant socio-economic thoughts and the present world. Free-market libertarianism with individualism remains strong in developed countries but is no longer compatible with the small earth. It has tremendous influences to underdeveloped countries, while there remain diverse cultures contradicting the free-market libertarianism. We discuss these features from the viewpoint of the present economics.

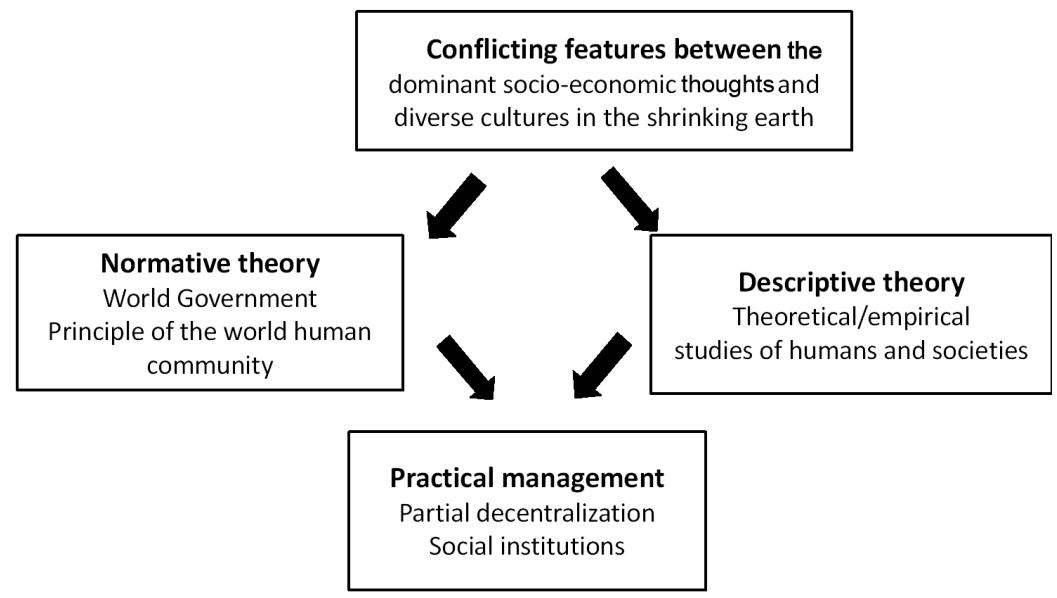

Figure 1. Logical Structures of the Themes. 
In Section 3, we consider the normative theory for the earth as unity. The Hobbesian social contract theory is applied to the entire world, where his "state of nature" is replaced by the total destruction of the earth. This change leads to a different conclusion that all people should be treated equally and freed from vested rights. We argue that this extreme view is evitable for the small earth but at the same time, it has great difficulties in its applications.

In addition to the normative theory, we need to reconsider the foundations and developments of human/social sciences in order to understand the present worldwide problems. In Section 4, we reconsider the standard economics and game theory from the methodological point of view. These fields are well developed but lack the reflective thoughts on their boundaries from the outside. We consider these features and give a brief description of a new theory called inductive game theory (Kaneko \& Matsui, 1999; Kaneko \& Kline, 2008), which is motivated by reflective thoughts on cognitive assumptions in the standard theories, i.e., bounded rationality and conservativeness.

To think about the problems of the entire world, we look at historical changes/developments of societies and social institutions. In the end of the $19^{\text {th }}$ century, which is only 120 years ago, the worlds and institutions tremendously differed from the present world. Vast changes have been occurring with social structures, but people with bounded logical and perceptional abilities were not able to follow such changes. In Section 5, we discuss needs of positive/empirical studies of societies and social institutions.

Even though we expect more technological developments, the basic nature of human beings will not change drastically. In Section 6, we argue that practical management of the earth should be decentralized, and here social sciences play crucial roles.

In Section 7, we conclude our explorations into new socio-economic thoughts on a small and narrow earth. As a whole, the article describes where the earth should go and what we, social scientists, should/could do, though these appear very difficult.

\section{Conflicting Features in a Small and Narrow Earth}

\subsection{Economics as a Science and a Thought}

Although economic activities are one among many social aspects of human worlds, they become more important as people pursue materially richer lives. This tendency is getting stronger as technology is progressing, with which the earth is even getting smaller and narrower. In this section, first, we look at how the present economics thinks, and also consider, from the viewpoint of the entire earth, what important issues are.

Economics has two sides; social science and social thought. The former is to study objectively the economic structures and behavior of economic agents. The latter includes some normative judgments, based on the former, about how society should be. The economics of the $20^{\text {th }}$ century emphasized its former side so 
that economics should be a value-free science; it should be silent of normative judgments. However, since it treats people and societies, we cannot avoid the questions of how human economic lives and societies should be. The present economics still keeps a tradition of this value-freeness, but has slightly retreated; instead of avoiding such normative questions, it looks for an economic science with minimum normative judgments.

Also, the present economics has a strong tendency to focus only on economic aspects as if all social aspects could be treated in the same manner. In this article, we will take the position that economic aspects are only part of the entire human worlds. From this perspective, we should critically investigate the foundations of the present economics. We now start with its benchmark.

\subsection{Theory of Perfect Competition}

The theory of perfect competition in the tradition from Adam Smith (1776) is suitable to the research attitude of minimizing normative judgments. The salient characteristic of the theory of perfect competition is to start with a very individualistic description of an economic agent (a consumer and/or a producer), restricting its scope only to economic aspects of human beings and societies. The theory as described in general equilibrium theory succeeds in explaining the behavior of the market economy as a harmonization of activities of many economic agents. See Hicks (1939), Debreu (1959), and Arrow \& Hahn (1971). This success, however, has made many economists ignore other aspects different from economic ones.

Game theory has been regarded as supplements for the theory of perfect competition to allow us more micro behavior of people and societies. John von Neumann, the founder of game theory, intended to surpass the idea of perfect competition, while including descriptions of other aspects of human beings and societies (cf. von Neumann, 1945, and von Neumann \& Morgenstern, 1944). However, his followers have not taken such steps toward a development of a new basic idea for the understanding of humans and society: At present, game theory is effectively included in economics and its teaching is not very different from the theory of perfect competition. In Section 3, we will discuss the necessity of a development in the direction von Neumann addressed. It will be presently argued that the theory of perfect competition is, in fact, used to support some social thought, but its basic structure leads people to unconsciousness of the social thought.

In the theory of perfect completion, given fixed prices, each consumer maximizes his utility and each firm maximizes its profits. The theory purports that a well-organized market institution guarantees commodities and services to be suitably produced and circulated. One main result, called the (first) fundamental theorem of welfare economics, (cf., Arrow \& Hahn, 1971), states that the resulting outcome of the market is optimal in the sense of Pareto. It means that the market functions with no waste in production processes as well as in distributions (exchanges) of commodities and services. 
This result is typically summarized as "optimization of economic efficiency by decentralization". It may be easier to divide this statement into three levels; the individual level, organizational level, and entire economy level. The first is a motivation for an individual agent (laborer, employer, or entrepreneur). The work environment for each agent should be designed to promote his/her work motivation. The second requires an economic organization (a private firm or a public sector) to be created so as to guarantee that each individual agent as well as the organization itself can pursue freely their profits/utilities and that efficiency is achieved for the organization as a result of their free behavior. The last level is that the total profits/utilities of the entire economy are maximized as a result of the free and competitive behavior of the economic agents and organizations in the economy.

For example, the privatization of the Japanese railway company divided into several independent companies, which took place in 1987, is based on the first two levels of the above idea to eliminate legal constraints on decision making by workers and organizations. The transition of former East-European communist countries into market economies, which started around 1990, is based more clearly on all the three levels of decentralization.

A basis of the idea of perfect competition is the large-number assumption that each economic agent at the individual or organizational level has many (or at least several) similar competitors. Under this assumption, each individual or organizational economic agent should be a price-taker (or environment-taker), and his/its pursuit of profits/utilities contributes for the entire social benefits and welfare. This is the teaching of the theory of perfect competition, specifically, the fundamental theorem of welfare economics.

\subsection{Free-Market Libertarianism (Individualism) as a Social Thought}

The theory of perfect competition supports what we call free-market libertarianism. This school of thought takes individual economic freedom as the supreme principle, and thus regards a socio-economic institution allowing every agent to freely pursue his economic profit/utility as an optimal one ${ }^{1}$. However, this already includes a normative judgment in that society should allow such freedom for each economic agent as much as possible and political/legal systems should be arranged to support this idea. Although it asserts to maximize individual freedom, some political and institutional arrangement of society, e.g., basic property rights, is inevitably needed.

Since a political institution finally chooses a social system, we should mention the political counterpart of free-market libertarianism. It is political libertarianism, which asserts a minimum political system to support individual freedom. These two thoughts appear to include no normative judgments, but make the normative judgment that such individualistic freedom has the supreme value.

${ }^{1}$ Pareto optimality allows many "optimal" states, and the theory of perfect competition chooses one or a few states among others. 
These include typically the presumption that each person's ownership including property rights is taken for granted. This is seriously problematic in the present small and narrow earth.

\subsection{Expansion of Human Activities in a Shrinking Earth}

Many problems in the present earth suggest us to rethink free-market libertarianism (and individualism). In the world, there are many societies with different cultural traits incompatible with free-market libertarianism, for example, family ties are still very important in many Asian countries. Religions are substantial part of society and human lives in the world, even in Europe and North America. These societal aspects constitute the substructures for economic lives and activities. Here, we postpone our discussions on societal and cultural aspects to Sections 4 and 5; instead, we look at free-market libertarianism from the viewpoint of economics itself taking the smallness and narrowness of the earth.

As mentioned above, free-market libertarianism relies upon the fundamental theorem of welfare economics. In fact, in addition to the large-number assumption, this theorem needs another assumption, which we call the large-environment assumption, that the natural environment behind the economy is large enough for economic activities to have only negligible influence to the environment. This assumption may already be inconsistent with the large-number assumption; that is, economic activities of a large number of economic agents almost necessarily change the environment. The fundamental theorem should be regarded as one (not really fundamental) theorem under these assumptions.

What we pointed out above is often called a market failure in the economics literature. However, the function of market as productions and exchanges is quite another problem. An example is an economy with air pollutions by automobiles; it is convenient for each economic agent to use a car, and even though an individual agent stops using a car, his contribution for an improvement of air is negligible in the city. Thus, each agent continues to use a car, and pollution also continues and may become serious. Unless the pollution escalates to the level of incurring serious damages to inhabitants, the market could still function to promote individual economic activities. The market itself generates and often magnifies the environmental problem. This structure exists commonly behind environmental problems such as global warming in the present earth.

Theoretical discussions and some examples on the above phenomena, widespread externalities, are given in Hammond, et al. (1989) and Kaneko \& Wooders (1994). Implications are extensively discussed in Kaneko (2004), $3^{\text {rd }}$ act.

It may help our understanding to give a brief look at the history of economics. Economics has a history of about 250 years from Adam Smith, and slightly more than 100 years from the beginning of the $20^{\text {th }}$ century when full-dress research was started. Now in the $21^{\text {st }}$ century, the influence of human activities is entirely different at the material and cognitive levels from 250 years, 100 years and even 50 years ago. The large-environment assumption might not be a problem in the 
time of Smith. However, if we carefully look at local events in the past, we find that the large-environment assumption was already inappropriate in many places. Various ancient civilizations collapsed by destroying their natural environments. In the $18^{\text {th }}$ and $19^{\text {th }}$ centuries, the American West was still a frontier, the natural environment there was almost unbounded relative to human activities, but in the old continents, a lot of famines and hungers happened constantly and victimized many people.

The above point is confirmed by looking at the world populations of the past and future. The world population exceeded 1 billion in the beginning of the $19^{\text {th }}$ century; it reached 3.0 billion in 1930 and 4.0 billion in 1965 . The present total human population on the earth is 7.2 billion in 2013, and according to the United Nation's prediction, the total human population of the world will reach 9.6 billion in the year $2050^{2}$. It would be enough to say that the total population of 2100 would be much beyond 10 billion. Taking these figures into account, the large-environment assumption is no longer valid; we cannot keep free-market libertarianism (and the fundamental theorem of welfare economics) as a sound basis of the socio-economic thought for the present-future earth.

\subsection{Globalization: Explosive Progress of Information and Transportation Technology}

Another important fact we should admit is the rapid progress in information and transportation technology. Scientific knowledge and technological progress are explosive as a whole, which may support globalization of economic and industrial activities. This makes the worldwide trades possible, but at the same time, it generates a lot of social problems; an obvious example, other than global warming, is the instability of the worldwide market, e.g., the 2008/2009 financial crisis. Some local event may influence the worldwide market so rapidly through information channels; this differs entirely from the world of 30 years ago. In fact, the concept of perfect competition requires also informational decentralization, and is not compatible with rapid spreads of information (cf., Kaneko, 2004: p.106). This requires us to rethink the present economics and game theory.

\subsection{Steady Slowness: Diversified Cultures and Values in the World}

We are apt to think that the entire world can and/or does move in the same direction by globalization and people will have the same and uniform values in the near future. This inclination is caused by the negligence of local social backgrounds surrounding each person: While accumulated knowledge and technology in the human world are getting larger, the ability of an individual being remains, more or less, unchanged. At the superficial level, technological progress will give more global information, but at a slightly deeper level, each person is created by his family and community in that he follows the patterns of behavior and thinking developed in his community and its local history. This locality

"World Population Prospects-Population Division-United Nations". esa.un.org. Retrieved 2016-09-15. 
leads to a great social diversity of cultures, languages, religions, values, etc. in the world, which together with globalization cause inevitable serious conflicts between groups of different cultures. This will be discussed in Section 4 from the viewpoint of inductive game theory and bounded rationality.

The above fact is confirmed by looking at the evidence that the worldwide illiteracy rate in 2015 is still more than 20\%, and that many developing nations have even higher illiteracy rates. This implies the existence of a big barrier to exclude illiterates from economic activities to be supposed in the present economics and game theory.

Thus, the present world shows explosive progress of informational technology, and only some people in some nations are enjoying prosperities brought about by such technological progress, sacrificing many other people and denying their cultures.

\subsection{Unity of the Earth vs. Diversity of Cultures in the Earth}

We are meeting various contradictory problems occurring in the earth. The one is that the earth is small and narrow with the growth of human population and explosive technological progress, which implies that individualism such as free-market libertarianism can no longer be a suitable social thought. In Section 3 , we discuss a normative theory for the future earth as unity, though we should admit a great variety of human cultures and difficulties in practical applications. The contradictory features may have no reconciliation, but at least we should look at them seriously.

\section{Normative Theory for the Earth as Unity}

\subsection{The Entire Earth as Unity}

There are about 200 sovereign nations in the present earth. It is an implication of the smallness and narrowness of the earth that those nations will no longer be able to retain their sovereignty in the near future. We will inevitably face some worldwide problems for which we should take the entire earth as unity. For example, when present nations face severe conflicts, or when a problem at the level of the entire earth happens such as global warming, we need to consider the integrated unity of the nations and people in the earth, which we call the world nation. We cannot directly go to practical management of the entire earth by the world nation. The earth has too many people and many local regions for practical managements. When an event is serious and cannot be resolved by local authorities, the world nation should intervene in the event. The world nation is the supreme authority to make a normative judgment for such an event ${ }^{3}$.

\footnotetext{
${ }^{3}$ This argument sounds related to "global justice" discussed in the field of philosophy of law. It has been discussed that developed countries are responsible to poverty in underdeveloped countries such as in Africa. The main issue in this field is to look for moral responsibilities of the advanced nations and their people. Many viewpoints have been discussed, but Rawls' (1971) distributive justice relative to "global justice" is the central term (see Pogge, 2001). We will discuss only briefly the relation of Rawls to the normative theory to be given in this paper.
} 
Since many local and global events possibly occur in the present and future earth, the normative theory for the world nation should have a scope large enough to evaluate each of possible events. For example, the scope should include genocides such as ones occurred in Cambodia of 1970's and the Congo area in 1990's, and famines and hungers occurring in the present sub-Saharan Africa. When the earth is running well, we do not need the normative theory; but we do need it when some very bad events happen or are expected to happen. To enable to have all possible events in the scope of the theory, we should choose the ultimate worst possibility for the entire earth and can discuss other events in a relativistic manner in the scope.

In fact, the problem of the ultimate worst possibility was already discussed by two great thinkers. They are the $17^{\text {th }}$ century philosopher, Thomas Hobbes, and the $20^{\text {th }}$ century physicist, Albert Einstein. Hobbes was the initiator of social-contract theory, and put the worst scenario as the basis for his social-contract theory of a nation. Einstein considered the total destruction of the earth by nuclear bombs as the worst possibility for the earth.

\subsection{Hobbes's Social-Contract Theory of a Nation}

Thomas Hobbes belonged to the $17^{\text {th }}$ century, and he mentioned nothing about the small and narrow earth and the world nation. Nevertheless, the logic of his social-contract theory of a nation described in his "Leviathan" (1651) can almost directly be extended into the social-contract theory of the world nation. In order to study the logical origin of a nation, he considered the hypothetical state of the society, called the "state of nature", by eliminating all social institutions and governmental authorities for protection of the individual rights from the present society. We emphasize that this is the logical origin of a nation but not a historical origin/emergence of a nation.

In the state of nature, since no authorities protect and control people's rights and duties, everybody owns the unbounded rights for everything. These rights contradict each other, and lead the state of nature to "a war of all against all", where everybody robs everybody else: Hobbes described the state of nature as "In such condition, there is no place for industry; ... no culture of the earth; ... no arts; no letters; ... and the life of man, solitary, poor, nasty, brutish, and short'. To avoid this cruel state, everybody gives up and provides almost all rights to the nation, and agrees on a social contract to have the central authority for governing the nation ${ }^{4}$.

\subsection{Einstein's Principle for World Peace}

In the paper on the special relativity theory published in 1907, Albert Einstein derived the conclusion that mass might be transformed into energy; if mass $m$ is

${ }^{4}$ Hobbes (1651) himself went to the conclusion of the "political absolutism" to centralize all the rights to the nation except for a small number of "natural rights" for individual members. The present author does not think that his conclusion is inevitable from his state of nature. 
changed into energy $E$, it obeys the formula $E=m c^{2}$, where $c$ is the speed of light and is gigantic (about $300,000 \mathrm{~km} / \mathrm{sec}$ ). Hence, if, even, a small amount of mass is changed into energy, the tremendous amount of energy would be released. In the 1940's, atomic bombs became technically possible, and in 1945, atomic bombs were thrown to Hiroshima and Nagasaki victimizing 300 400 thousands of people. In the 1950's, the USA and USSR (the present Russia) already kept a sufficient number of atomic (hydrogen) bombs to destroy the entire earth.

In the 1940's, Einstein recognized the crisis of the earth, and faced the fact that the earth was no longer unbounded for human beings. Being apprehensive of human race in the earth, Einstein wrote in a letter to a Russian scientist in 1949:

\section{4. (*): The Objective of Avoiding Total Destruction (of the Earth) Must Have Priority over Any Other Objective ${ }^{5}$}

This has been called the principle for world peace.

It is to avoid the worst possibility for the earth and human race. The worst possibility differs from Hobbes's time in that human beings can now destroy the entire earth. In our normative theory, we consider problems and event in the present/future earth from this point of view. It is time for us to think about the very existence of the entire earth and human race in order to look at worldwide events. Taking this extreme reference point, we can think about any events to possibly occur in the present/future earth in a relativistic manner.

\subsection{The Hobbes-Einstein-Nash Social-Contract Theory of the World Nation}

After the finding of possible release of atomic energy, the implication of Hobbes's question of what would happen if no social institutions guarantee individual rights. In Hobbes's age, its logical/physical implication is "a war of all against all". However, now, the pure logical answer to his question is the total destruction of the earth and annihilation of the human race.

Our normative theory for the world nation is obtained from the Hobbes's social-contract theory by substituting the destruction/annihilation of the earth and human race for Hobbes's state of nature. Substitution of the total destruction for the Hobbesian state of nature has an implication very different from Hobbes's as well as from currently prevailing thoughts about rights and duties for an individual being. To understand this implication, we look at the nature of vested rights in the context of free-market libertarianism.

\subsection{Abrogation of Vested Rights and Interests}

Property rights are very basic for libertarianism; ownerships for properties (and, more basically, individual lives) are protected by a law and police power from stealing, robbing, and killing. This legal and police power needs to be financed

${ }^{5}$ Einstein (1968: p. 146). 
by a tax system, which is a partial denial of property rights; and this system is needed for free-market libertarianism to work.

We, living in "modern" developed societies, are apt to think that ownership of some property should belong to its owner, and that each individual should have integrity, meaning that his/her identity belongs to himself/herself. For this, some social systems are needed to protect ownerships and individual integrity. Many of us living in developed societies have not experienced states without such protecting social systems. Similar beliefs are held on "sovereignty" of a nation meaning that it should retain its own rights and interests. Once, however, a nation is in a war or is beaten by another nation, individual integrity and nation's sovereignty are meaningless.

If we take individual integrity and a nation's sovereignty literally, people in developed nations could have no duties/rights and ignore droughts, famines, wars, and genocides in Africa. A cause for droughts and famines may be economic activities in developed nations. People in the developed nations think that they should give some aids to the people suffering from droughts and famines ${ }^{6}$. This gives only a temporal resolution, and is not more than an adjustment of the free-market libertarianism.

Very contrary to the free-market libertarianism, in the Hobbes-Einstein social-contract theory for the world nation, no individual beings and no nations can keep vested rights. From the viewpoint of $\left(^{*}\right)$, we reconsider social institutions and events as possible alternative targets but not as granted. For practical managements of the earth, we may need social systems allowing property rights to some extent, but we are now considering a basis for evaluations of social systems for such managements.

\subsection{The Principle of the World Human Community}

The principle $\left(^{*}\right)$ is more extreme than Hobbes's state of nature; it is applied to the very existence of each individual being. From the viewpoint of $\left(^{*}\right)$, each human being and his rights exist simultaneously with the entire earth. This is formulated as follows:

\section{8. (**WHC) The Body and Talent of Each Human Being Belong to the World Human Community, Which Owns All Rights for Them. The World Human Community Consists of All Human Beings on the Earth}

All rights including those to his body and talent are taken away from each but are gathered to the world human community. The world human community itself is a hypothetical entity, and it may be regarded as already presumed for the principle $\left(^{*}\right)$. The principle ( ${ }^{*}$ WHC) itself, however, induces no conclusive decisions. We assume the other principle for the world human community to make decisions:

${ }^{6}$ Arguments of this type have been found in the field of global justice (see Pogge, 2001). 


\section{9. (**IRW) Every Individual Being in the Community Has the Entire Right to the World human Community}

We denote these two, $\left({ }^{* *} \mathrm{WHC}\right)$ and $\left({ }^{* *} \mathrm{IRW}\right)$, simply by $\left(^{* *}\right)$. We call this $\left({ }^{* *}\right)$ the principle of the world human community.

The principle $\left.{ }^{* *}\right)$ has a similar status to Hobbes's state of nature. In either $\left.{ }^{* *}\right)$ or Hobbes's, each being has the entire rights, but in Hobbes, physical/technological constraints lead to "a war of all against all". In our case, the development of atomic bombs leads to the total destruction of the earth, and the property rights of each is entirely denied, including the body and talent. Hobbes's argument is still logically consistent; "a war of all against all" shows it, the avoidance of which leads to a social contract for the nation. On the other hand, in $\left.{ }^{* *}\right)$, each retains all rights even to the entire earth. When at least one disagrees, he can destroy the earth, which means that each keeps the switch of the huge atomic bomb.

The principle ${ }^{* *}$ ) denies and abrogates all the vested interests and rights of all people and nations. This is the supreme normative principle for individual rights. Practically, we cannot directly follow ${ }^{* *}$; when we need the very basis of our normative principle, we should recall this principle.

Let us apply the principle $\left({ }^{*}\right)$ to people waiting for death by famines, hungers, or genocides in Africa. It is legitimate for the suffering people to demand people in the developed nations to save them. The people in developed nations cannot ignore those demands; it is their duty to save the suffering people by sacrificing (some part of) their rich lives, since not only the economic capitals of developed nations but also the bodies and talents of people there belong to the world human community. It is a more apparent duty for all the people in the world to immediately stop genocides. Neither sovereignty nor the "nonintervention of the affairs of other nations" can be applied here.

\subsection{The Nash Social Welfare Function}

A mathematical formulation of the Hobbes-Einstein social contract theory is given as the theory of the Nash social welfare function proposed in Kaneko \& Nakamura (1979). The Nash social welfare function is given as

$$
W(x)=\sum_{i=1}^{n} \log \left(u_{i}(x)-u_{i}\left(x_{0}\right)\right),
$$

where $i=1, \cdots, n$ are the members of the world nation, $u_{i}$ is the utility function of member $i$ in the sense of expected utility theory, $x$ is a world state to be evaluated, and $x_{0}$ is the total destruction/annihilation of the earth and human race.

Mathematically speaking, the Nash social welfare function of (1) is a different representation of an $n$-person version of the Nash bargaining solution given by Nash (1950). It is the main difference that in the former, the disagreement point is the total destruction of the earth, while in the latter, the disagreement point is given or determined in each situation. 
By associating the Nash bargaining theory with the Nash social welfare function, the social-contract of our normative theory becomes more explicit and is described as follows. Everybody has a button to destroy the earth, and only decision is made when it reaches a unanimous agreement. Thus, everybody makes a decision on agreeing or not, taking into account the possibility of his disagreement to lead to the total destruction of the earth. Here, nobody can guarantee his vested rights and interests ${ }^{7,8}$.

Let us apply the Nash social welfare function to the above mentioned problems of famines/hungers/genocides. The world state $x$ includes the deaths of suffering people in the near future. For any person $i$ of these people, this state $x$ is very close to the total destruction $x_{0}$. Although the utility $u_{i}(x)$ is still higher than $u_{i}\left(x_{0}\right)$, the utility difference $u_{i}(x)-u_{i}\left(x_{0}\right)$ is positive but close to 0 , equivalently, $\log \left(u_{i}(x)-u_{i}\left(x_{0}\right)\right)$ is almost $-\infty$. This means that the total social welfare $W(x)$ is also almost $-\infty$. Hence, the Nash social welfare function suggests to avoid the world state $x$ and choose any world state $y$ in order to stop deaths from famines/hungers/genocides, for example, by sacrificing rich lives of people in developed nations.

\subsection{Difficulties in Applications of the Principle of World Human Community}

We have a lot of difficulties in practical applications of the principle of the world human community ${ }^{* *}$ ) or the Nash social welfare function to more general and less extreme social problems. In the above example, the lives/deaths of some people are compared with the total destruction/annihilation of the earth and human race. In this case, the physiological part of "utility" dominates the social/cultural part. In less extreme problems, however, "utility" is closely related to society and culture; in many cases, "utility" is formed by interactions with society. Red sweaters may give higher utilities to some people than blue ones, for example, because red is the symbol color of their community. In this example, social-psychological part becomes dominant than physiological part. A utility loss by taking blue sweaters is negligible to that of genocide or starvation. Psychological "utility" is not as important as death or lives, and even may not need to be counted in social welfare. We, as social scientists, should study the problems of what "utility" is and when it should be taken into account seriously".

Direct and immediate actions are required in the example of famines/hungers/ genocides. These involve effectively no time structures. In many other social

${ }^{7}$ This argument may look similar to some studies of contractarian-morality such as in Binmore (1994), Gauthier (1986) in that agreement is the fundamental components for morality. However, our normative theory is not to explore people's (natural) morality, which belongs to the subjects in Section 4 , but we take a different view for morality.

${ }^{8}$ The above argument requires stringent mathematical foundations, and indeed, such mathematical foundations must be revealed to see the scope and limitations of this theory. Those mathematical discussions should be given in a separate paper.

${ }^{9}$ In the literature of social choice and welfare, it was asked whether or not individual welfare (utility) is a right foundation for social welfare. Also, it is asked whether social decision processes should be taken into account. See Suzumura (2011). 
problems, however, we cannot directly choose resulting states; instead, we should use some social institutions to manage societies. Therefore, social institutions and their management become the target of our study, which will be discussed in Sections 4 and 5. Together with such studies, we may apply our normative theory to the choice of social institutions.

\subsection{Right-Based Normative Theory}

The reader might wonder why we do not adopt Rawls' (1971) theory of distributive justice. This theory starts with the social choice behind the veil of ignorance and measuring in the worst position in the society. This is interpreted as expressing a unanimity decision of the members of the community in question; in this sense, it is claimed that the theory is contractarian. The veil of ignorance argument is more coherent to Harsanyi's (1953) utilitarianism than Rawls (1971), as far as we look at them from the viewpoint of the present utility theory. Nevertheless, these theories have no scopes to include the entire earth, nor no further consideration of individual rights is given. On the other hand, our normative theory, as in the Hobbesian theory, is based on the hypothetical setting to eliminate the institutions protecting and controlling individual rights from every member of the world. Thus, our theory is a right-based normative theory.

As above mentioned, the use of social institutions will be inevitable for practical management of the earth. The central target of a design of a social institution is to control individual rights, within which each individual is admitted to have freedom of behavior and thinking. Our theory evaluates a design of distributions of individual rights from the viewpoint of the principle of the world human community $\left.{ }^{* *}\right)$.

\section{Theoretical Studies of Humans and Societies}

Here, we claim that a majority of individual beings are effectively living in their local communities and are unchanged or changing slowly, even while they are surrounded by globalized economic activities. This claim implies a great difficulty in practical management of the entire earth. The claim itself is not new, and is found in some social sciences such as sociology and cultural anthropology. But we need a theoretical investigation of it in order to have a better understanding of the human world. The key theory is inductive game theory recently developed by the present author and his collaborators (e.g., Kaneko \& Matsui, 1999; Kaneko \& Kline, 2008 $)^{10}$. In this section, we explore the claim and its implications to the small and narrow earth.

\subsection{Diversity of Customs, Behaviors, and Ways of Thinking}

Some reader may doubt the above claim, since convenient and rapid informational technology will be available to people, with which they may communicate to more people in the local and global manners. We argue below that the social

\footnotetext{
${ }^{10}$ The basic research program is described in Kaneko (2004).
} 
context surrounding them exceeds their cognitive abilities. A change in informational technology is limited to the superficial level; communication device relies upon a language, and a language is too coarse to convey their deeper and detailed cultural backgrounds.

Let us give a rough sketch of the idea of inductive game theory. It is a basic observation that people cannot instantaneously adapt themselves to new situations. No a priori knowledge on the situation is available to a newcomer. Situational knowledge is only obtained by putting themselves to a new situation and having experiences there. Learning from experiences requires a lot of trials/errors; each time, a person may learn only small part of the structure. To understand the structure better, he needs to put him deeply to the situation and experience the same situation many times. However, experiences are scattered over time, and forgetfulness may prevent from recalling them.

These activities are all time consuming and constrained to local social environments and individuals' cognitive and perceptional abilities. These constraints lead to inevitable slowness of individual changes. The reader may reflect upon his social network and how many people he can communicate to in his local community. This observation together with a large society has the implication that cultures, behaviors, and ways of thinkings are diversified even with rapid information technology. Such diversity was emphasized by Benedict (1934), a cultural anthropologist, by observing many advanced and primitive societies. She was strongly against the biological-environmental determinism of human cultures that natural, biological, and environmental components determine people's behavior and thinking.

\subsection{The Cognizance Assumption in the Standard Theories}

The above view is very different from the standard economics and game theory. It is typically and implicitly assumed in these fields that an individual agent has well-formed beliefs over the structure of the model; "a belief" is expressed as subjective probability in the sense of Savage (1954). This needs the cognizance assumption that the structure of the target situation is a priori known to each agent. The term "uncertain" which is often used as synonymous to "unknown" means that some estimated probability is less than 1, or more generally, there are multiple possibilities for given information. This requires the cognizance assumption that the set of possibilities is a priori known to an agent.

\subsection{Inductive Game Theory: Exploring Experiential Sources of Beliefs/Knowledge}

Instead of starting with the above cognizance assumption, inductive game theory explores the origin/emergence of beliefs/knowledge of the basic structure in individual experiences ${ }^{11}$. This changes our understanding of the social world by

${ }^{11}$ Here, the concepts of "belief" and/or "knowledge" are entirely different from those in the sense of subjective probability. It is about a symbolic sentence or symbolic structure. "Knowledge" is a true "belief", where truth is defined outside. See Kaneko (2002: Section 6). 
almost $180^{\circ}$ degree. First, let us see how inductive game theory thinks about society.

Each person is involved in various social situations of the social-web; families, friends, schools, offices, companies, etc. In Figure 2 , each of $\Gamma^{0}, \Gamma^{1}, \cdots$ is a social situation which may or may not involve him. The social-web is already very complex for him, and only some small part is directly related to him; the other parts are typically invisible to him. He follows some behavioral patterns in those situations. He has no a priori knowledge of the structure of each situation involving him. Only after experiencing a situation several or many times, he may have a view about the situation. Then he may choose a better alternative action using his understanding of the situation. If a situation is the first time for him, he can choose only a default action predetermined.

Once the above idea is taken, we would notice that the cognizance assumption for each agent is highly problematic. For example, let $\Gamma^{0}$ be the 2-person extensive game described in the left of Figure 3, which is described from the objective point of view: On the other hand, we make the basic assumption that neither of persons PL1 and PL2 knows the structure of the game. To have a clear distinction from the standard theory, let us assume that PL1 does not know even the existence of PL2 moving after PL1.

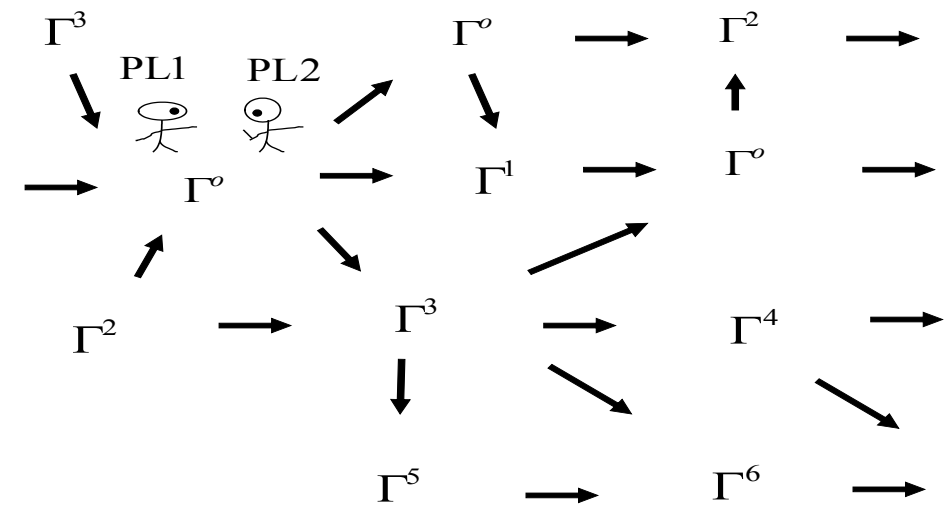

Figure 2. Social-Webb.

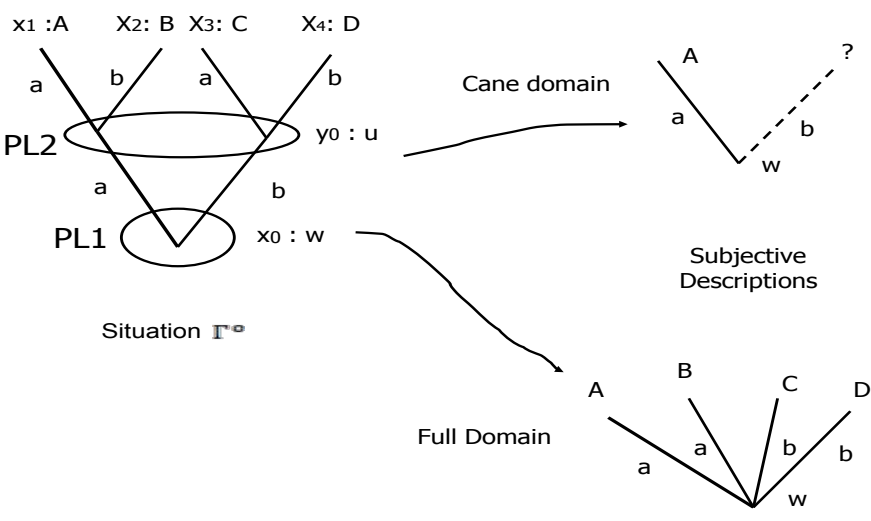

Figure 3. Objective Description and Subjective Understanding. 
When both PL1 and PL2 have always been playing act a, PL1's experiences give a view like the upper right tree having the outcome $A$ resulting from act $a$, while he recognizes another available act $b$ without knowing the resulting outcome from $b$. If each of PL1 and PL2 has tried both actions, then PL1 has experienced the four outcomes which, objectively, have been led by his two actions and PL2's two actions. Since PL1 does not know the existence of PL2, PL1 thinks that $A$ and $B$ are caused by his choice $a$, and similarly, $C$ and $D$ are by $b$. His view becomes like the bottom right tree; here, causality is not unique. Changing the assumptions on trials/errors and accumulated experiences, we have other possible individual views.

The above game is extremely simple; yet we meet such multiplicity of individual views. For a game with more people and more available actions, the number of possibilities becomes astronomical. One society consists of a huge and complex social-web, and people belonging to different parts of the social-web have developed their own views.

The computerized technology does not help very much each person to learn details of the social-web. This constraint will not be eliminated by progress in technology, since complexity involved is astronomical and the people have bounded cognitive and perceptional abilities ${ }^{12}$.

The above view is consistent with Benedict (1934) against the biological-environmental determinism of human cultures. Theoretically speaking, we can study how such diversity has originated and emerged. Within the theory, however, it would be difficult to find which forms of cultures occur more likely; the theory can talk about the structure but may not find detailed patterns in diversity. To see detailed patterns, we need to look at real worlds and empirical research is required. Such studies should compensate for the theory, which will be discussed in Section 5.

\subsection{Underlying Model of a Human Being: Conservative Nature}

An individual person typically follows his behavior pattern and only occasionally makes deviations from it. In addition, a person has also a patterned way of thinking. These are because making trials/errors is behaviorally and mentally costly. In a social context, in addition, he may have learned patterned behavior and thinking from other people from his childhood.

The underlying model of a human being in inductive game theory is such a pattern-governed person, but only occasionally, he thinks about new possibilities. New trials are scarce at the behavioral and mental level for each being. Thus, a human is necessarily conservative for his present situation and thought. This gives a clear distinction from the standard economics and game theory, which will be discussed now.

\footnotetext{
${ }^{12}$ Recently, we have certain relevant developments. Kline, Lavendhomme, \& Waltener (2018) describe a constructive process of a derived view from experiences. Kaneko (2017) gives a constructive expected utility theory by restricting the permissible probabilities to finite (decimal) fractions. These suggest a systematic study of the concepts of induction, deduction, and bounded rationality.
} 


\subsection{Cognizance Assumption in the Standard Economics and Game Theory}

It is the cognizance assumption for the standard theory that an individual agent has well-formed beliefs over the structure of the model. This has various derivative aspects: for example, utility maximization is regarded as an important component of it, and the cognizance assumption is a sufficient condition for utility maximization, but it is often ignored how utility maximization reaches a maximizing point.

A related aspect is the overuse of set-theoretical apparatus. All symbolic propositional expressions are represented by sets of possibilities. The cognizance assumption is based on this. For example, the concept of information is expressed as a set of alternative possibilities. In real world, however, information comes to a person as a language (symbolic) expression. Expressing it as a set of possibilities requires a set-theoretical interpretation of symbolic expressions. This is also a base for an instantaneous utility maximization.

\subsection{Methodological/Philosophical Studies}

Here, we mention necessity of methodological (/philosophical) studies of our research itself. As we need a normative theory to discuss objectives and evaluations, methodological studies may direct theoretical and empirical research; without such bases, theoretical research could become mere mathematical exercises, and empirical research might be simple pursuits of social events.

In "Nineteen Eighty-Four", Orwell (1949) wrote about a new system of language called the "new speak" forced upon people. Behind the new speak is the philosophical idea that if some words were removed from the dictionary, the concepts described by them would be eliminated. For example, if the word "revolution" were removed from the dictionary, then people might no longer be able to conceive of a revolution. This metaphor is effectively observable in our profession. Here, we give two examples among others; Arrow's (1951) impossibility theorem and free use of the "probability" and "subjective probability".

Arrow's impossibility theorem is regarded as an important theorem in welfare economics, claiming that it is impossible to find a social welfare function satisfying certain "plausible" conditions. One interpretation of a social welfare function typically adopted is a mechanism of aggregating the preference relations reported by the members of society. We find a difficulty in this interpretation in that "having a preference relation" should be distinguished from "knowing his own preference relation". The former is already problematic but not very serious. On the other hand, the latter is deeply problematic: How does one know his own preference relation? If the number of alternative choices is two or three, it would not be problematic, but in the case with more alternatives, it is difficult to think about his own preference relation. This is compared with a statement about one's brain; it is unavoidable to assume that a person has a brain but cannot know its inner functioning. 
Another example is free use of "probability" and/or "subjective probability". People's beliefs are expressed by "subjective probability" by Savage (1954). In the theory of games with incomplete information from Harsanyi (1967-'68), "subjective probability" is extensively used expressing beliefs about nature as well as other's beliefs including logical inferences. Savage (1954) gave an axiomatization of "subjective probability", but any "axiomatization" is not beyond necessary and sufficient conditions; some premathematical argument or evaluation about the suitability of those conditions should be given. In the case of "subjective probability", the real problem is where and how such a probability comes from ${ }^{13}$. The use of such beliefs expressing logical inferences is just coming from ignoring the other field of mathematical logic, which has a long history from the ancient Greece as a scientific study of human logical inferences.

\subsection{Necessity of New Theories}

The above are examples of existing theories regarded as important in the economics and game theory. Although the initiators of those theories dig new problems at their times, these theories are now mediocre. According to Thomas Kuhn (1964), a history of science has a long steady period of progress generating variants within one paradigm and has a possible scientific revolution only when serious anomalies appear.

Now, the earth and human race are in the urgent situation; we cannot wait for a long period before a revolution in our sciences. Instead, we should be conscious about what an important problem is and what a mediocre one is. We should attack the very heart of each problem.

\section{Descriptive/Empirical Studies of Societies and Institutions}

People from different communities meet each other more in the future earth; their meetings inevitably causes frictions and conflicts between those people. Since different backgrounds are deeply impregnate in different communities, diversity cannot be easily erased by teaching a common language such as English and/or computer technology. In this section, we will discuss what kind of research is required to understand such diversified cultures.

\subsection{Geological and Historical Understanding of the Human World}

First of all, we need to investigate what events have occurred, are occurring, and will possibly occur in the human world. There are two kinds, horizontal and vertical, of empirical research for these questions: the horizontal research is to study what are occurring over the present earth, and the vertical one is to study what

\footnotetext{
${ }^{13}$ In the first half of the $20^{\text {th }}$ century, the question of what "probability" is was discussed an extensively. Among various interpretations, the frequentist interpretation is only an attempt to view "probability" from the material world. See Weatherford (1982). For expected utility theory, von Neumann \& Morgenstern (1944), Chap.1 explicitly stated that they adopt the frequentist interpretation. See $\mathrm{Hu}$ (2013) for a treatment of expected utility theory from the frequentist perspective.
} 
have occurred in the past of each region. To consider the future world, we need these different methods.

From the horizontal point of view, we see a great variety of human cultures as well as events occurring in the present world. To study people and their behaviors in different situations helps our understanding of human beings and societies. On the other hand, from the vertical point of view, we trace events in the past of a region and understand what social structures and social institutions lead to them. By those studies, we may foresee possible events to happen in the future earth and find some ways to prevent them.

\subsection{Historical Investigations of Japanese Societies}

Kaneko (2007) adopted the vertical methodology to look into Japanese histories of 400 years. Even though Japan is geologically small, its histories are rich and give hints to consider the future earth and possibility of the world nation. The modern Japan started in 1868, and before it, Japan was governed by the Tokugawa family, a feudal clan, for the period from 1603 to 1867: during this period, Japan had the policy of national isolation from 1633 until 1858, except for one channel (a small island in Kyushu) to the Netherlands. After 1868, Japan was quickly industrialized, and took the militarism until the end of World War II. Then, it has grown and become one of the richest nations in the world.

Looking into Japanese societies in the past, we find some social situations hardly perceivable in the present Japan. For example, extreme poverty prevailed in the rural areas in the Tohoku ${ }^{14}$ region in Japan from the Tokugawa period until some years after the end of World War II. This is just one history relevant to our considerations. In the following, we mention some other observations about Japanese histories, which give a hint to understand market economies.

\subsection{Concentration of Land Ownerships and Its Implications}

In the Japanese histories, we find that private ownership, which is one of our crucial target issues as already discussed, created a small number of huge landlords and a large number of peasants. Some peasants were observed in the Tokugawa era, but after the reform of the tax system on farm lands in 1873 (6 year after the Meiji restoration), the percentage of peasants in the Tohoku region had increased from $15 \%-20 \%$ to $45 \%-50 \%$ in 40 years. Suffering cold weather for several years, many peasants in despair sold their daughters for prostitution in Tokyo. After World War II, the concentration of land ownership was fully dissolved by the allied forces. In fact, this concentration of land ownerships and political dissolution were repeated many times from the civilization in Japan (about $7^{\text {th }}-8^{\text {th }}$ century).

The market system with the specific legal structure led to the above mentioned concentration of land ownerships. As discussed in Section 2, the central assumption for the market system is property rights (private ownerships), which needs a legal protection. For the practical management of the future earth, it could be

${ }^{14}$ Tohoku is the north east area of the main island of Japan. 
inevitable to use a market system in a certain manner, which requires us to admit property rights. However, we should always be careful of controlling the system of property rights ${ }^{15}$.

\subsection{Conservativeness and Its Implications}

Concentrations of land ownerships are social phenomena. Scrutinizing, however, into landlords' behavior and speaking, we find very conservative nature of their mentality; they were apt to think that their ownerships would be eternal. Their justification often took the following form; their furthest ancestors reclaimed wastelands by hard work and all successors had kept their lands from generations to generations. Some bought lands from other farmers, but they were all legally legitimate.

Their attitudes may be called rationalization. This is consistent with what we discussed in Section 4; with a great multiplicity in possible individual views derived from an individual's experiences, we have a tendency to find one convenient explanation (cf., Kaneko \& Matsui, 1999). It is also important to notice that such an explanation is often based upon some convenient part of reality. This is the conservative nature of a human being. Our normative theory states that we should abrogate vested rights and interests, but in reality, people naturally want to keep them; they are conservative, regardless of being conscious or unconscious. Therefore, the principle of the world human community, denying them entirely, is needed to make us conscious of our conservative nature.

\subsection{Administrative Units in the Past}

Before 1868, Japan was divided into more than 300 feudal states and people were not able to across freely the boarders between those states. Poor people such as farmers were strictly restricted to their local places, and their views were limited to their places. Therefore, each state developed its dialect and culture. After the Meiji restoration, Japan became one nation without legal boundaries, but people from different states had difficulties to communicate. This was true until recently between people even from next states, but now young people, due to the TV, speak the standard Tokyo dialect. This fact may tell that diversity may be getting smaller in Japan.

It is relevant here that the number of feudal states in 140 years ago was more than 300 , and before 1868 , the scope of each person was typically restricted to his small state. But now, nobody thinks that each feudal state is his country. This change has happened only in 140 years ${ }^{16}$. But it is unclear that diversity of people

\footnotetext{
${ }^{15}$ Piketty (2013) shows, using data of incomes in many countries, concentrations of wealth through market economies, and argues the necessity of high marginal income tax rates up to $80 \%$.

${ }^{16}$ Another striking fact is that the total population in Japan did not grow for the period of the Tokugawa era. It grew from 1600 to 1700 , but after it, the total population declined for many years, and only at the end of the Tokugawa era, the total population was rebounded to reach the same level as that of 1700 . This fact was not caused by a high rate of infant mortality; the birth rate itself was law during the period from 1700 to 1867. After the Meiji restoration, the birth rate became high. Some demographic anthropologists have worked but did not give a good explanation for such decline of population.
} 
is getting smaller at least at a non-superficial level.

\section{Management of the Future Earth}

We have discussed the necessity of the world nation for the future earth, but also argued that the earth would remain fulfilled with diversified cultures. Each individual being has a small social world, while being connected to larger societies in various ways from social institutions and legal structures such as family, schools, companies, towns, prefectures, and nations. Without those institutions, we could not keep our present societies, economic activities, and people's welfare. In 2,400 years ago, Plato (cf. the republic, 1955) already gave comprehensive discussions on the needs of social institutions to run a nation.

Social institutions are connected in complicated manners; some are hierarchical, some are nested, exclusive, or inclusive. For example, a private company, which is a relatively small social institution, can take economic activities freely under the protections of local or central governments, which are larger social institution, provided that the company pays legitimate taxes.

The above description is more or less the same in many nations in the present world. However, when worldwide disasters happen in some places in the earth, sovereignties of involved nations should no longer be kept; and the world nation should play roles of stopping and/or coordinating. To control worldwide problems such as global warming, over-population, etc., coordination between the individual economic activity level and worldwide global level would be more difficult.

One possibility is to return to the control of all detailed activities of local community or even every individual person. This is, more or less, the communist planning economy, where the central planner makes all detailed production plans. The failure of this idea was historically shown by communist nations up to 1990. Once a great diversity of cultures is recognized, this failure could be logically clear. Therefore, some decentralized managements of political units and economies are inevitable. Again, we meet the use of democracy and market systems. But as already discussed, we cannot go to free-market libertarianism without restrictions. Now, we can say only that a restrictive use of a market system is aimed for practical management, but how to be restrictive must be our future study.

\section{Larger Administrative Units: Contradictory Features}

As stated in Section 5, until 140 years ago, the Tokugawa family acted as the central government but Japan remained divided into more than 300 feudal states as administrative units. In the present European Union, the borders are practically removed, and people can move freely in search of a job from one nation to any other within the Union. In the year 2100, the concept of a "nation" will be less clear-cut in the European Union.

The above description of the historical direction may be interpreted as mean- 
ing that the unification of present nations to a certain degree would be a natural conclusion. Also, some centralized management would be needed, but it is subject to risks of global instability, meaning that centralization may magnify an external shock and lead to a worldwide disaster. On the other hand, we have discussed also that due to diversified societies, decentralization of economic management is inevitable. Thus, we have a lot of contradictory features for practical management of the entire earth. We should study how to reconcile the centralized idea of the world nation with practical decentralization.

\section{Conclusion}

In this article, we considered various problems expected for a small and narrow earth of the future earth. Our discourse consisted of two almost contradictory parts: a normative theory and a descriptive theory. The former provides evaluations of possible events and of designs of social institutions from the viewpoint of the earth as unity. The latter, discussed as social sciences for practical management of the earth, states that a great diversity of cultures will remain, which implies that a unified management of the earth is practically very difficult.

The latter requires us to do a lot of theoretical and empirical studies in comprehensive manners. In particular, we discussed inductive game theory, but it is still premature and needs to be developed in many ways. Also, for a historical study, we looked briefly at the Japanese societies of past 400 years. It gave a dynamical change of the social structure of Japan, and perhaps a similar change could be observed in the histories of other countries. This may give a lot of hints for further considerations of the future earth. In this article, we have not discussed geological differences in the world. Such differences should be considered empirically as well as theoretically.

We, social scientists as well as other scientists, should now very seriously investigate the problems of the future earth. To make a meaningful recommendation, we need to understand the world better, which requires philosophical, theoretical, and empirical studies. These should include technical studies of the external worlds, people's internal mental worlds, normative studies, and philosophical studies of researchers' methods and their attitudes. We are also required to synthesize studies of different fields; in the present academia, various fields on close subjects exist almost separately. Scientists should cooperate to save the earth and human beings. As our task is urgent, we should take a truly radical attitude for every step.

\section{Acknowledgements}

The author thanks Jeffrey J. Kline, Nathan Berg, Yao Zeng, Oliver Schulte, and the referee of this journal for many helpful comments on earlier drafts. Also, he thanks various people including Koichi Suga for helpful comments. He is partially supported by Grant-in-Aids for Scientific Research No.26780127 and No.17H02258, Ministry of Education, Science and Culture. 


\section{References}

Arrow, K. J. (1951). Social Choice and Individual Value. New Haven, CT: Yale University Press.

Arrow, K. J., \& Hahn, F. H. (1971). General Competitive Analysis. San Francisco, CA: Holden-Day.

Benedict, R. F. (1934). Patterns of Culture. New York, NY: Mariner Books.

Binmore, K. (1994). Game Theory and the Social Contract (Vol. 1). Cambridge, MT: VMIT Press.

Debreu, G. (1959). Theory of Value: An Axiomatic Analysis of Economic Equilibrium. New Haven, CT: Cowles Foundation Monographs Series.

Einstein, A. (1968). Ideas and Opinions. New York, NY: Bonanza Books.

Gauthier, D. (1986). Morals by Agreement. Oxford: Clarendon Press.

Hammond, P. J., Kaneko, M., \& Wooers, M. H. (1989). Continuum Economies with Finite Coalitions: Core, Equilibria and Widespread Externalities. Journal of Economic Theory, 49, 113-134. https://doi.org/10.1016/0022-0531(89)90070-7

Harsanyi, J. C. (1953). Cardinal Utility in Welfare Economics and in the Theory of Risk Taking. Journal of Political Economy, 61, 434-435. https://doi.org/10.1086/257416

Harsanyi, J. C. (1967-68). Games with Incomplete Information Played by Bayesian Player. Management Science, 14, 159-182, 320-334, 486-502.

Hicks, J. R. (1939). A Value and Capital. Oxford: Oxford University Press.

Hobbes, T. (1991: The Original 1651). Leviathan. Cambridge, MT: Cambridge University Press.

Hu, T.-W. (2013). Expected Utility Theory from the Frequentist Perspective. Economic Theory, 53, 9-25. https://doi.org/10.1007/s00199-009-0482-9

Kaneko, M. (2002). Epistemic Logics and Their Game Theoretical Applications: Introduction. Economic Theory, 19, 7-62. https://doi.org/10.1007/s001990100202

Kaneko, M. (2004). Game Theory and Mutual Misunderstanding. Heidelberg: Springer Verlag.

Kaneko, M. (2007). Social Justice-Considered in Hell. Tokyo: Keiso-shobo. (In Japanese)

Kaneko, M. (2017). Expected Utility Theory with Probability Grids and Incomparabilties. Working Paper No. E1610, Waseda University. https://www.waseda.jp/fpse/winpec/assets/uploads/2014/05/No.E1610Kaneko.pdf

Kaneko, M., \& Kline, J. J. (2008). Inductive Game Theory: A Basic Scenario. Journal Mathematical Economics, 44, 1332-1363. https://doi.org/10.1016/j.jmateco.2008.07.009

Kaneko, M., \& Matsui, A. (1999). Inductive Game Theory: Discrimination and Prejudices. Journal of Public Economic Theory, 1, 101-137. https://doi.org/10.1111/1097-3923.00005

Kaneko, M., \& Nakamura, K. (1979). The Nash Social Welfare Function. Econometrica, 47, 423-435. https://doi.org/10.2307/1914191

Kaneko, M., \& Wooders, M. H. (1994). Widespread Externalities and Perfectly Competitive Markets: Examples. In R. Gilles, \& P. Ruyes (Eds.), Imperfection and Behavior in Economic Organizations (pp. 71-87). Amsterdam: Kluwer Academic Publisher.

Kline, J. J., Lavendhomme, T., \& Waltener, S. (2018). From Memories to Inductively Derived Views: A Constructive Approach. To appear in Economic Theory.

Kuhn, T. S. (1964). The Structure of Scientific Revolutions. Chicago, IL: Chicago University, Press. 
Nash, J. F. (1950). Two-Person Bargaining Game. Econometrica, 18, 155-162. https://doi.org/10.2307/1907266

Orwell, G. (1949). Nineteen Eighty-Four. New York, NY: New American Library.

Piketty, T. (2013). Capital in the Twenty-First Century. Cambridge, MT: Belknap Press. https://doi.org/10.4159/9780674369542

Plato (1955). The Republic. Translated by Lee D., London: Penguin Books.

Pogge, T. W. (2001). Global Justice. London: Blackwell.

Rawls, J. (1971). A Theory of Justice. Cambridge, MT: Harvard University Press.

Savage, L. J. (1954). The Foundations of Statistics. New York, NY: John Wiley and Sons.

Smith, A. (2017, Original 1776). An Inquiry into the Nature and Causes of the Wealth of Nations. Amsterdam: Metalibrary.

Suzumura, K. (2011). Introduction Chapter: Handbook of Social Choice and Welfare (Vol. 1, pp. 1-26). Amsterdam: North-Holland.

von Neumann, J. (1945, Original 1938). A Model of General Equilibrium. Review of Economic Studies, 13, 1-9.

von Neumann, J., \& Morgenstern, O. (1944). Theory of Games and Economic Behavior. Princeton, NJ: Princeton University Press.

Weatherford, R. (1982). Philosophical Foundations of Probability Theory. Routledge \& Kegan Paul. 